\title{
Article
}

\section{Reversible Lectin Binding to Glycan-Functionalized Graphene}

\author{
Tereza Koukalová ${ }^{1,2,3}$, Petr Kovaříček ${ }^{1,2, *}$ (D) Pavla Bojarová ${ }^{3,4, *(\mathbb{D})}$, Valentino L. P. Guerra ${ }^{1,2}$ (D), \\ Vladimír Vrkoslav ${ }^{5}$, Lukáš Navara ${ }^{1}$, Ivan Jirka ${ }^{1} \mathbb{D}$, Marek Cebecauer ${ }^{1} \mathbb{D}$, Vladimír Křen ${ }^{3} \mathbb{D}$ and Martin Kalbáč ${ }^{1} \mathbb{D}$ \\ 1 J. Heyrovsky Institute of Physical Chemistry of the Czech Academy of Sciences, Dolejškova 2155/3, \\ 18223 Prague, Czech Republic; koukalot@vscht.cz (T.K.); guerrav@vscht.cz (V.L.P.G.); \\ lukannavara@gmail.com (L.N.); ivan.jirka@jh-inst.cas.cz (I.J.); marek.cebecauer@jh-inst.cas.cz (M.C.); \\ martin.kalbac@jh-inst.cas.cz (M.K.) \\ 2 Department of Organic Chemistry, Faculty of Chemical Technology, University of Chemistry and \\ Technology Prague, Technická 5, 16628 Prague, Czech Republic \\ 3 Institute of Microbiology of the Czech Academy of Sciences, Vídeňská 1083, 14220 Prague, Czech Republic; \\ kren@biomed.cas.cz \\ 4 Department of Health Care Disciplines and Population Protection, Faculty of Biomedical Engineering, \\ Czech Technical University in Prague, Nám. Sítná 3105, 27201 Kladno, Czech Republic \\ 5 Institute of Organic Chemistry and Biochemistry of the Czech Academy of Sciences, \\ Flemingovo Náměstí 542/2, 16610 Praha, Czech Republic; vladimir.vrkoslav@uochb.cas.cz \\ * Correspondence: petr.kovaricek@vscht.cz (P.K.); bojarova@biomed.cas.cz (P.B.)
}

Citation: Koukalová, T.; Kovaříček, P.; Bojarová, P.; Guerra, V.L.P.;

Vrkoslav, V.; Navara, L.; Jirka, I.; Cebecauer, M.; Křen, V.; Kalbáč, M. Reversible Lectin Binding to Glycan-Functionalized Graphene. Int. J. Mol. Sci. 2021, 22, 6661. https:// doi.org/10.3390/ijms22136661

Academic Editor: Cheorl-Ho Kim

Received: 27 May 2021

Accepted: 16 June 2021

Published: 22 June 2021

Publisher's Note: MDPI stays neutral with regard to jurisdictional claims in published maps and institutional affiliations.

Copyright: (c) 2021 by the authors. Licensee MDPI, Basel, Switzerland. This article is an open access article distributed under the terms and conditions of the Creative Commons Attribution (CC BY) license (https:/ / creativecommons.org/licenses/by/ $4.0 /)$.

\begin{abstract}
The monolayer character of two-dimensional materials predestines them for application as active layers of sensors. However, their inherent high sensitivity is always accompanied by a low selectivity. Chemical functionalization of two-dimensional materials has emerged as a promising way to overcome the selectivity issues. Here, we demonstrate efficient graphene functionalization with carbohydrate ligands-chitooligomers, which bind proteins of the lectin family with high selectivity. Successful grafting of a chitooligomer library was thoroughly characterized, and glycan binding to wheat germ agglutinin was studied by a series of methods. The results demonstrate that the protein quaternary structure remains intact after binding to the functionalized graphene, and that the lectin can be liberated from the surface by the addition of a binding competitor. The chemoenzymatic assay with a horseradish peroxidase conjugate also confirmed the intact catalytic properties of the enzyme. The present approach thus paves the way towards graphene-based sensors for carbohydrate-lectin binding.
\end{abstract}

Keywords: graphene; wheat germ agglutinin; carbohydrate; 2D materials; sensor

\section{Introduction}

Two-dimensional (2D) materials hold a great potential for application as the active layers of sensors due to their strict monolayer character. Graphene, the epitomical example of a 2D family of materials, can achieve sensitivity down to single atoms under particular conditions [1]. However, the great sensitivity of graphene is compensated by its poor selectivity, which hampers the straightforward transfer of the technology to practice. The cornerstone of graphene-based sensor development is thus to achieve selective recognition of a given analyte [2,3].

Lectins are a broad family of proteins [4,5], featuring a carbohydrate recognition domain (CRD) that binds sugar moieties with a high specificity. Lectins play diverse roles in biological systems; they participate in cellular signaling, are involved in biochemical pathways leading to various pathologies (cancer [6], arthritis [7], etc.), and are essential in cell-cell recognition in infectious diseases such as AIDS [8,9], tuberculosis [10], and even the SARS-CoV-2 virus [11]. Lectins have been shown to form complex quaternary structures, ranging from dimers to higher homo- or heterooligomers up to chimeric structures that can gradually interchange [12,13]. This challenge is further complicated by the agglutinating properties of lectins and the chemical resemblance of their carbohydrate ligands. 
Monolayer graphene functionalized with specific carbohydrate ligands can serve as a suitable active sensing layer for detecting lectin activity [14]. When placed into ionic solutions, it forms an electric double layer of ions on its surface [15-18]. Protein binding changes the Stern layer composition, which can be used as a functional principle of a sensor. In this way, highly specific graphene-based sensors of various lectin proteins can be constructed and used in biochemical and biomedical research and clinical practice. However, the major challenge of achieving the specific lectin-carbohydrate binding and distinguishing it from a non-specific interaction remains [19-21].

In this work, we demonstrate the specific binding of wheat germ agglutinin (WGA) to monolayer graphene covalently functionalized with $\mathrm{N}$-acetylglucosamine (GlcNAc) chitooligomers of varying lengths. WGA is a $34 \mathrm{kDa}$ homodimeric lectin with a total of eight carbohydrate binding sites [22], which features a high affinity to chitooligomers composed of several subunits of GlcNAc. We used a series of techniques [23] to characterize functionalization up to the reversible WGA binding in unprecedented detail. The data unambiguously confirm the scheme of chemical transformations taking place on the $2 \mathrm{D}$ monolayer. Ligand competition studies confirm the higher avidities of WGA for longer chitooligomers [24] and, therefore, the specificity of interactions on the functionalized surface, which is the crucial step in the development of graphene-based lectin sensors.

\section{Materials and Methods}

\subsection{Synthesis of Functionalized Chitooligomers}

For the preparation of 2-azidoethyl-functionalized chitooligosaccharides composed of $\beta(1 \rightarrow 4)$-bound GlcNAc units (Scheme 1 ), we used a one-step transglycosylation reaction catalyzed by the Tyr470Asn mutant of the $\beta-N$-acetylhexosaminidase from Talaromyces flavus, which we had developed previously [25]. In this reaction, 2-azidoethyl 2 -acetamido-2-deoxy- $\beta$-D-glucopyranoside (GlcNAc-O-EtN $\mathrm{E}_{3}$; mono-GlcNAc), prepared chemically [24], was used as a glycosyl acceptor. 2-Azidoethyl 2-acetamido-2-deoxy- $\beta$-Dglucopyranosyl-( $1 \rightarrow 4)$-2-acetamido-2-deoxy- $\beta$-D-glucopyranoside (di-GlcNAc), 2 -azidoet hyl 2-acetamido-2-deoxy- $\beta$-D-glucopyranosyl-( $1 \rightarrow 4)$-2-acetamido-2-deoxy- $\beta$-D-glucopyran osyl-(1 $\rightarrow 4$ )-2-acetamido-2-deoxy- $\beta$-D-glucopyranoside (tri-GlcNAc), 2-azidoethyl 2-acetam ido-2-deoxy- $\beta$-D-glucopyranosyl-( $1 \rightarrow 4)$-2-acetamido-2-deoxy- $\beta$-D-glucopyranosyl-( $1 \rightarrow 4$ )2 -acetamido-2-deoxy- $\beta$-D-glucopyranosyl-( $1 \rightarrow 4)$-2-acetamido-2-deoxy- $\beta$-D-glucopyrano side (tetra-GlcNAc), and 2-azidoethyl 2-acetamido-2-deoxy- $\beta$-D-glucopyranosyl-(1 $\rightarrow 4)$-2acetamido-2-deoxy- $\beta$-D-glucopyranosyl- $(1 \rightarrow 4)$-2-acetamido-2-deoxy- $\beta$-D-glucopyranosyl$(1 \rightarrow 4)$-2-acetamido-2-deoxy- $\beta$-D-glucopyranosyl-( $1 \rightarrow 4)$-2-acetamido-2-deoxy- $\beta$-D-glucop yranoside (penta-GlcNAc) were then prepared by transglycosylation reaction based on a previously reported procedure [24]. The structural characterization data (NMR, HRMS) of compounds mono- to penta-GlcNAc are fully in accordance with the literature [24].

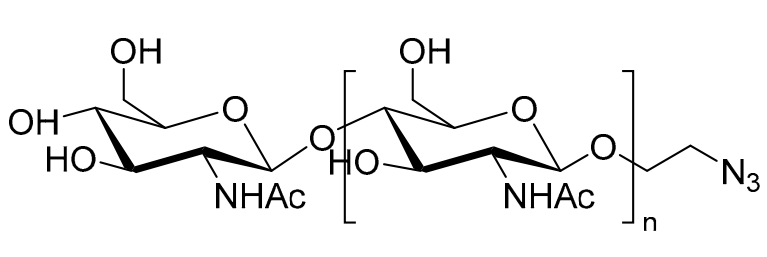

$$
\mathrm{n}=\left\{\begin{array}{l}
0=\text { mono-GIcNAc } \\
1=\text { di-GlcNAc } \\
2=\text { tri-GIcNAc } \\
3=\text { tetra-GIcNAc } \\
4=\text { penta-GIcNAc }
\end{array}\right.
$$

Scheme 1. Structures of the GlcNAc chitooligomers used in this study.

\subsection{Graphene Synthesis and Functionalization}

Graphene was synthesized by the chemical vapor deposition method on a copper foil, transferred onto silicon chips with a $300 \mathrm{~nm}$-thick $\mathrm{SiO}_{2}$ layer by the copper etching/polymer-assisted method, and fluorinated as described earlier [26,27]. The terminal alkyne was introduced by nucleophilic substitution of fluorine by propargyl amine 
deposited on the chip as a $50 \%$ solution in $\mathrm{N}, \mathrm{N}$-dimethylformamide at room temperature for two hours and then washed with a large excess of methanol.

\subsection{The Cu-Catalyzed Alkyne-Azide (CuAAC) Reaction}

GlcNAc-O-EtN ${ }_{3}$ ligand $(2 \mathrm{mg})$ was dissolved in $\mathrm{CuSO}_{4}$ solution $(900 \mu \mathrm{L}, 100 \mu \mathrm{M})$ and mixed with sodium ascorbate solution $(50 \mu \mathrm{L}, 20 \mathrm{mM}$ ), and tris(3-hydroxypropyltriazol ylmethyl)amine solution $(50 \mu \mathrm{L}, 6 \mathrm{mM})$-all aqueous. This mixture was dropped onto the propargylated graphene chip ( $c a 1 \times 1 \mathrm{~cm}^{2}, c a 100 \mu \mathrm{L}$ per chip) and reacted at room temperature. After $30 \mathrm{~min}$, an additional $50 \mu \mathrm{L}$ of $20 \mathrm{mM}$ sodium ascorbate solution was dropped onto each chip and reacted for an additional $90 \mathrm{~min}$. All samples were then thoroughly washed with deionized water and air dried.

\subsection{Incubation with WGA and Competitor}

Wheat germ agglutinin (WGA, from Triticum vulgaris, Sigma-Aldrich, St. Louis, MI, USA) was dissolved in phosphate-buffered saline (PBS) at an approximate concentration of $1 \mathrm{mg} / \mathrm{mL}$, and $100 \mu \mathrm{L}$ of this solution was dropped onto each chip $\left(\mathrm{ca} 1 \times 1 \mathrm{~cm}^{2}\right.$ ). Incubation was performed at room temperature for $2 \mathrm{~h}$, and the samples were then washed with PBS buffer. Competitive unbinding of WGA was performed using GlcNAc solutions of defined concentrations in PBS at room temperature for $2 \mathrm{~h}$ followed by washing with PBS and deionized water.

\section{Results and Discussion}

Monolayer graphene is rather inert and aggressive reagents are required for its activation (Figure 1). We used the previously reported fluorination with $\mathrm{XeF}_{2}$ in the gas phase at room temperature under reduced pressure [28,29]. The initial high fluorine content (ca 40 atomic \% with respect to carbon) decreased within two days to an equilibrium value of about 10 atomic \% [29], which means that every C-F bond is surrounded by nine sp ${ }^{2}$ carbon atoms on average. This arrangement renders the fluorine atom relatively easily exchangeable for suitable nucleophiles [28,30], such as the amino group of propargyl amine. Thus, the monolayer graphene may efficiently be grafted with terminal alkynes. In the next step, chitooligomers of different lengths carrying an azidoethyl substituent at the anomeric carbon were attached to the graphene surface using the standard CuAAC click reaction protocol in water [31]. Finally, specific binding between chitooligomer ligands and the corresponding lectin (WGA) occurred in aqueous PBS. The multistep functionalization scheme is shown in Figure 1.

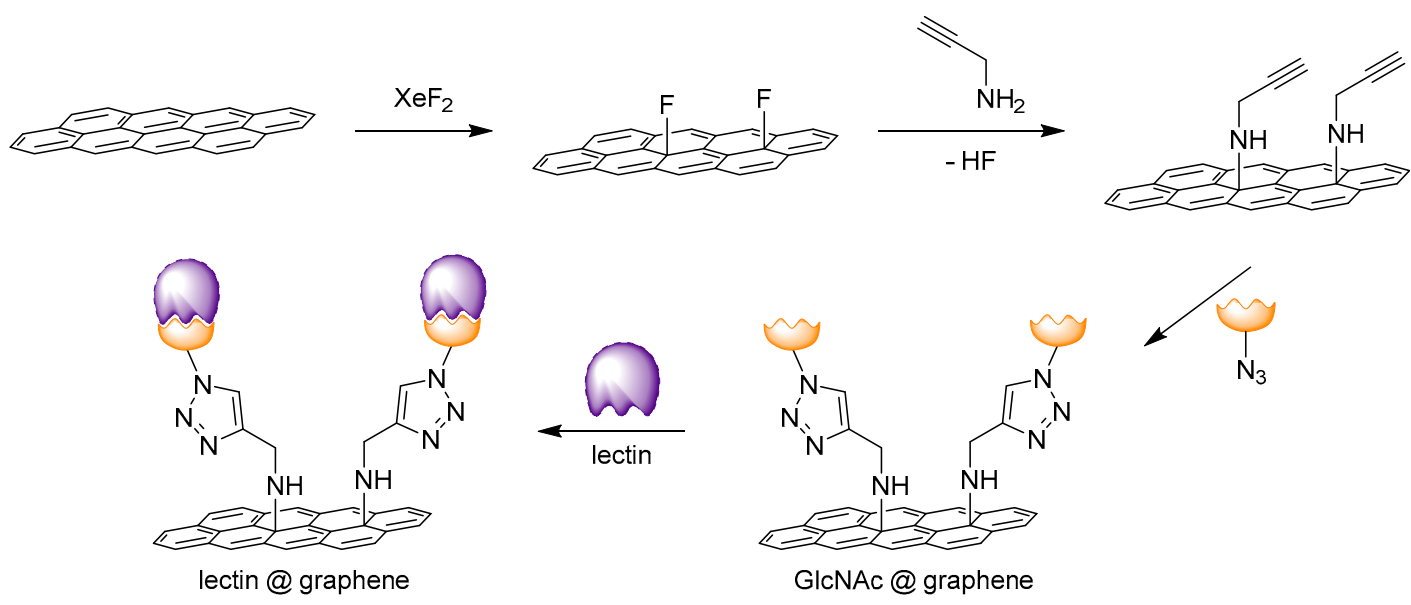

Figure 1. Scheme of the graphene chemical functionalization with chitooligomers for WGA binding. In the first step, pristine graphene was fluorinated, followed by nucleophilic exchange with propargyl amine. Using the CuAAC click reaction, azidoethyl-substituted chitooligomers $(\mathrm{GlcNAc})_{1-5}$ were grafted on the functionalized graphene. WGA lectin binds to chitooligomer ligands immobilized on the graphene surface. 
The process of graphene functionalization was monitored after each step by several methods in order to unambiguously prove the successful grafting of the desired species according to the designed scheme. Graphene on the $\mathrm{Si} / \mathrm{SiO}_{2}$ substrate is slightly hydrophilic [32,33], with a water contact angle (CA, Figure 2$)$ of $79.7( \pm 1.9)^{\circ}$. Fluorination and propargylation led to negligible changes in the contact angle, with values approaching $80.0( \pm 3.4)^{\circ}$ and $82.3( \pm 1.4)^{\circ}$, respectively. In contrast, a large difference in hydrophilicity was observed after the click reaction with chitooligomers-a single GlcNAc unit (monoGlcNAc) decreased the contact angle to $70.5( \pm 3.8)^{\circ}$, while a di-GlcNAc ligand decreased it to $59.9( \pm 2.5)^{\circ}$. For longer chitooligomers, the CA value did not change any further and fell within a range of $60^{\circ}-65^{\circ}$. Atomic force microscopy (AFM, Figure 2, see the Supplementary Materials file) confirmed the expected changes by providing complementary information on the layer thickness measured at the edge of the graphene sheet after each step. The pristine transferred graphene thickness was $0.7 \mathrm{~nm}$. It increased to $0.9 \mathrm{~nm}$ after fluorination and to $1.7 \mathrm{~nm}$ after propargylation. Such an increase in thickness is in good agreement with the particular bond lengths $(\mathrm{C}-\mathrm{F}, \mathrm{C}-\mathrm{N}$, and $\mathrm{C}-\mathrm{C})$ in the grafted species ${ }^{28}$. After the reaction with chitooligomers, the layer thickness gradually increased along with the number of GlcNAc units, reaching a plateau at around $2.5 \mathrm{~nm}$ for the tri-GlcNAc ligand. Again, this value agrees with the $\mathrm{CA}$ values discussed above and the expected folding of longer flexible oligosaccharide chains on the surface. Importantly, AFM analysis of graphene decorated with the series of chitooligomers (from mono- to penta-GlcNAc) after incubation with WGA in PBS showed a uniform layer thickness of 5-6 nm, which is the estimated size of the $34 \mathrm{kDa}$ globular WGA protein. These results thus support the hypothesis that chitooligomer ligands on graphene bind to WGA binding sites and also rule out the possibility of multiple-layer non-specific aggregation. The summary of CA and AFM data is provided in Figure 2.

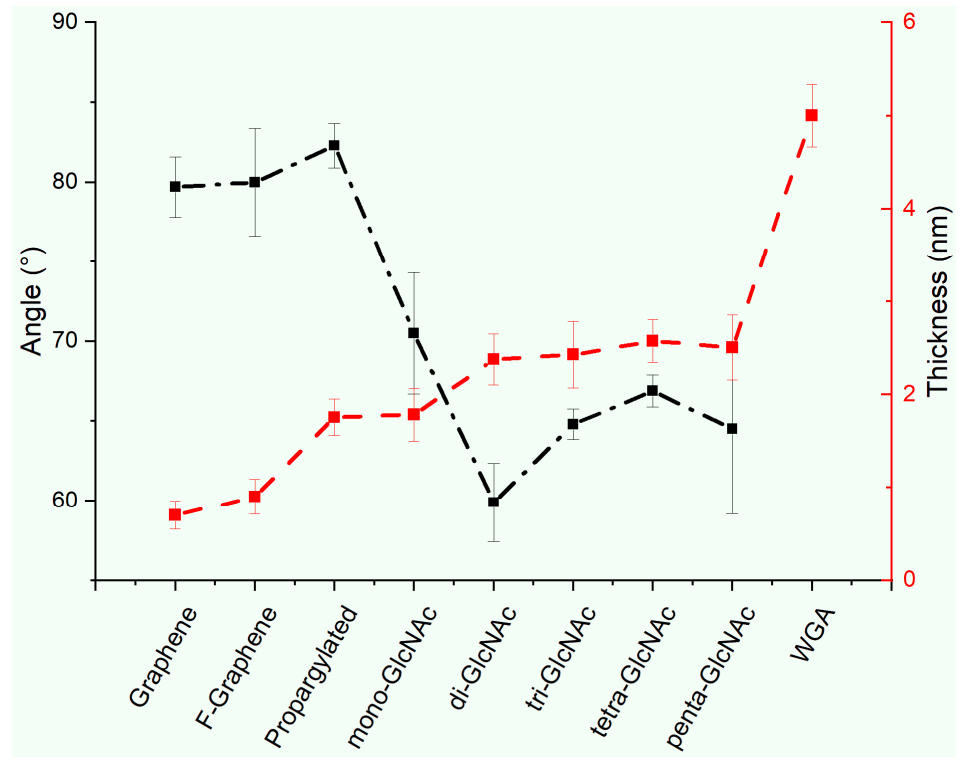

Figure 2. Summary of the AFM and CA data for graphene functionalization. AFM shows a gradual increase in the layer thickness with increasing size of the grafted species. Longer chitooligomers tended to fold back; hence, the thickness reached a plateau for tri- to penta-GlcNAc species. Importantly, after incubation with WGA, all chitooligomers showed a constant thickness of about $5 \mathrm{~nm}$. Correspondingly, the CA decreased when hydrophilic carbohydrates were grafted onto the graphene surface, and a plateau of between $60^{\circ}$ and $65^{\circ}$ was reached for tri-GlcNAc and longer chitooligomers. See the Supplementary Materials for definitions of error bars.

The structural integrity of chitooligomers grafted to the surface was confirmed by surface-enhanced Raman spectroscopy (SERS, Figure 3). Reference spectra of the chi- 
tooligomers were measured in the solid state, showing sharp peaks at expected positions (partial assignment for mono-GlcNAc is given in the caption of Figure 3). With the increasing number of units, the bands broadened due to minor differences in the surrounding particular functional groups for longer chitooligomers, making the spectra non-informative for tri-, tetra-, and penta-GlcNAc ligands (see Supplementary Figure S1). Graphene samples after the click reaction were covered by a $12.5 \mathrm{~nm}$-thick evaporated silver film, and Raman spectra were measured with a $633 \mathrm{~nm}$ excitation laser at intensities of $1-10 \mu \mathrm{W}$, focused with a $100 \times$ objective at a spot of about $1 \mu \mathrm{m}$. The laser intensity was carefully optimized because the thermal plasmon decay leads to overheating in the irradiated spot and to the degradation of the sample. The obtained SERS spectra matched well with the reference spectra, showing, for example, the characteristic amide I vibration at $1631 \mathrm{~cm}^{-1}$, $\mathrm{CH}_{2}$ deformation at $1473 \mathrm{~cm}^{-1}, \mathrm{C}-\mathrm{H}$ and $\mathrm{C}-\mathrm{OH}$ deformation at $1128 \mathrm{~cm}^{-1}$, and anomeric $\mathrm{C}-\mathrm{H}$ deformation at $866 \mathrm{~cm}^{-1}$ (Figure 3, assignment according to the literature $[34,35]$ ). It is important to note that the enhancement of particular bands in SERS strongly depends on the orientation of the molecular vibration with respect to the plasmon field, as it has been shown previously [36]. Therefore, relative band intensities between conventional and surface-enhanced Raman spectra cannot be directly compared.

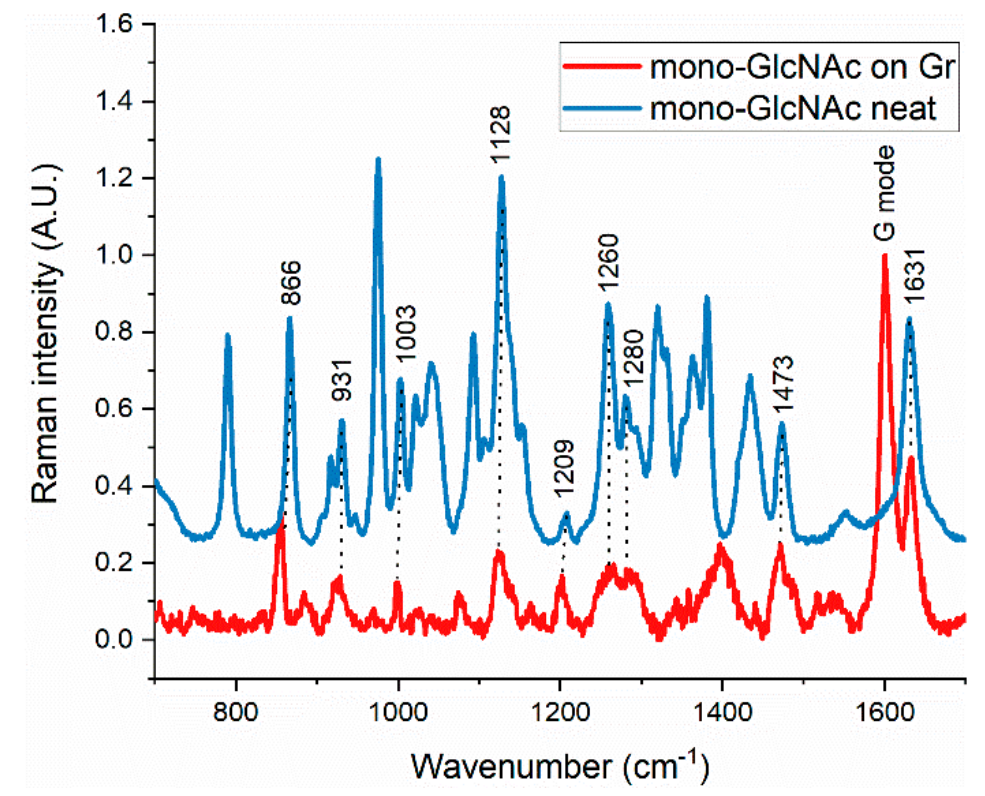

Figure 3. Comparison of Raman and surface-enhanced Raman spectra of mono-GlcNAc neat (blue) and grafted on graphene (red), respectively. Specific vibrations enhanced in the SERS mode are indicated in the spectra, e.g., amide I at $1631 \mathrm{~cm}^{-1}, \mathrm{CH}_{2}$ deformation at $1473 \mathrm{~cm}^{-1}$, and $\mathrm{C}-\mathrm{H}$ and $\mathrm{C}-\mathrm{OH}$ deformation at $1128 \mathrm{~cm}^{-1}$. The signal assignment was performed according to the literature [34,35].

To determine the interaction of lectin with chitooligomers, WGA was incubated with the GlcNAc-functionalized graphene for two hours at room temperature in PBS buffer. The successful binding was evaluated by matrix-assisted laser desorption/ionization mass spectrometry (MALDI) using sinapinic acid as the matrix. Functionalized graphene samples after incubation with either WGA and the reference WGA stock solution, deposited on silicon chips, both provided three dominant peaks at 8.5, 17.1, and $34.3 \mathrm{kDa}$ (Figure 4a). The highest value corresponds to the natural WGA dimeric form, the middle value corresponds to the polypeptide monomer, and the lowest mass is the doubly charged monomer.

The MALDI results were further corroborated by fluorescence imaging. FITC-labeled WGA was incubated with functionalized graphene samples deposited on glass coverslips, excited using the $488 \mathrm{~nm}$ laser, and detected on a sensitive EM-CCD camera. Specific binding of FITC-WGA to GlcNAc-functionalized graphene was clearly detectable at the 
graphene edge as an intense contrast line (Figure $4 \mathrm{~b}$ ). Finally, the binding was confirmed by a chemoenzymatic assay. WGA conjugated with horseradish peroxidase (WGA-HRP, along with non-conjugated WGA as a negative control) was immersed into the tetramethylbenzidine (TMB) substrate solution. Within seconds, the characteristic blue color of the oxidized TMB developed in the sample with WGA-HRP but not in the negative control (see Supplementary Figure S3).
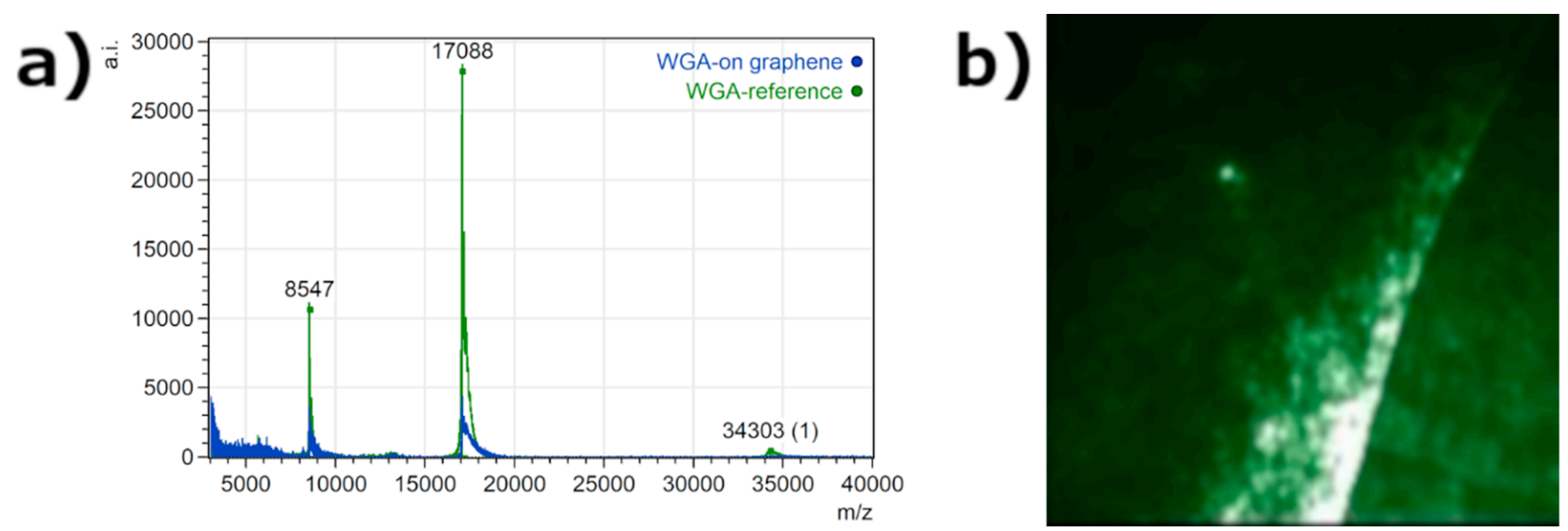

Figure 4. (a) MALDI spectra overlaying the reference spectrum of WGA (green), and the spectrum acquired on functionalized graphene after incubation with WGA (blue). The dominant masses in both spectra are 8.5, 17.1, and 34.3 kDa. (b) Fluorescence imaging of a graphene edge functionalized with GlcNAc after incubation with fluorescently labeled (fluorescein isothiocyanate, FITC) WGA. A clear contrast is observed at the edge of functionalized graphene.

An important feature of carbohydrate binding to lectins is the previously shown affinity increase [24] by approximately one order of magnitude when extending the chitooligomer by a single GlcNAc unit. We thus performed a binding assay in which WGA bound to GlcNAc-functionalized graphene was competitively released from the surface by the addition of the monomeric ligand in the solution (see also Supplementary Figure S2). Figure 5 summarizes the AFM measurement of the layer thickness of graphene decorated with mono- or di-GlcNAc on the surface after competition with a gradually increasing concentration of mono-GlcNAc in solution. At low concentrations of the mono-GlcNAc competitor, the layer thickness showed constant values of 5.5-6 nm, indicating the presence of WGA bound to the surface. At a mono-GlcNAc concentration of about $10^{-8} \mathrm{M}$, the layer thickness suddenly dropped to values corresponding to the functionalized graphene without WGA. The physical nature of AFM measurement did not allow us to obtain experimental points in the descending slope of the sigmoidal curve. The experimental data were fitted with the Hill function with a fixed Hill coefficient of 1.75 (positive cooperative binding) based on previous studies ${ }^{37}$ and provided dissociation constants of $7.3 \times 10^{-9}$ and $3.4 \times 10^{-8} \mathrm{M}$ for mono- and di-GlcNAc-functionalized graphene, respectively. For tri-GlcNAc and longer chitooligomers grafted on graphene, the competition with mono-GlcNAc did not proceed even at high competitor concentrations $\left(10^{-3} \mathrm{M}\right.$ GlcNAc), indicating that mono-GlcNAc is not an efficient competitor for tri- and longer chitooligomers immobilized on the graphene surface. 


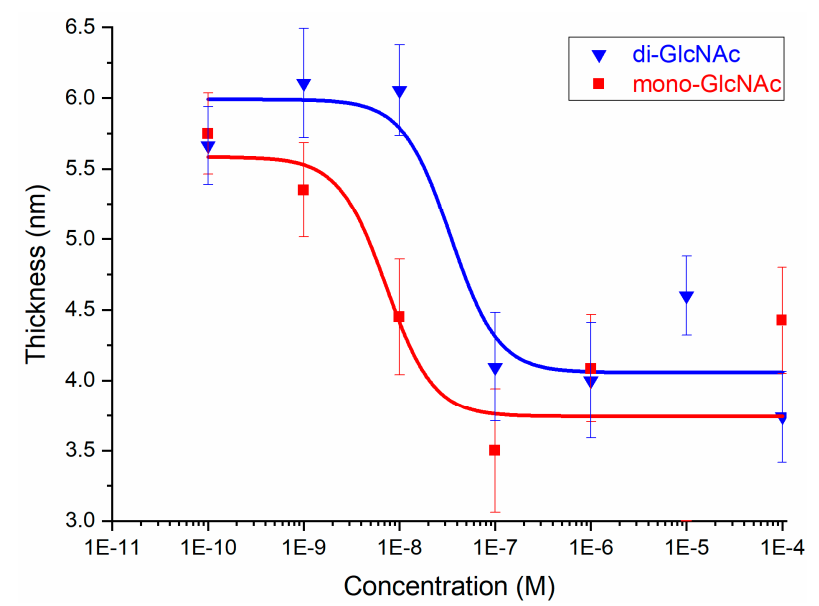

Figure 5. Comparison of the WGA binding curves obtained by AFM for mono- and di-GlcNAcgrafted graphene when in competition with free mono-GlcNAc ligand in solution. The curves were fitted with the Hill function with a fixed Hill coefficient of 1.75, as given in the literature [37]. The height drop for di-GlcNAc-grafted graphene occurred at about a five times higher competitor concentration than for the mono-GlcNAc-grafted graphene. The dependence of the dissociation constant on the chitooligomer length confirms the specificity of carbohydrate-lectin binding.

\section{Conclusions}

Monolayer graphene was functionalized with $\beta(1-4)$-linked $N$-acetylglucosamine chitooligomers of varying lengths. Chitooligomers feature a high affinity for wheat germ agglutinin, a homodimeric lectin with eight carbohydrate binding sites. The successful functionalization of the graphene surface was confirmed by a set of complementary techniques including Raman, AFM, CA, SERS, fluorescence microscopy, and mass spectrometry. The critical issue of the binding specificity between lectin and chitooligomer ligands exposed on the graphene surface was investigated in a competitive binding assay using AFM as the monitoring technique. The extensive characterization consistently proved the mechanism of graphene functionalization and the specific binding of WGA to the carbohydrates grafted on the functionalized graphene. This study thus paves the way towards specific sensors for the quantitative determination of lectins, in diverse solutes, produced by industry or the natural environment.

Supplementary Materials: The following are available online at https:/ /www.mdpi.com/article/10 .3390/ijms22136661/s1: Additional spectra, images, data tables, and instrumentation descriptions.

Author Contributions: T.K., P.K., and V.L.P.G. conducted graphene synthesis, transfer, and functionalization. P.B. and V.K. synthesized and characterized the chitooligomers. V.V. conducted MALDI-MS experiments. L.N., T.K., and V.L.P.G. conducted Raman and AFM measurements. I.J. conducted contact angle measurements. M.C. conducted fluorescence imaging. P.K. wrote the manuscript. M.K. gathered grants that have been paying for the work that we have done, participated on text editing and corrections. All authors participated in data analysis and editing of the manuscript. All authors have read and agreed to the published version of the manuscript.

Funding: This work was supported by project No. 18-09055Y awarded by the Czech Science Foundation, and by the mobility projects LTC20072 (COST Action CA18103 INNOGLY) and LTAUSA19001 by the Ministry of Education, Youth and Sports of the Czech Republic. This work was further supported by the European Regional Development Fund; OP RDE; Project: "Carbon allotropes with rationalized nanointerfaces and nanolinks for environmental and biomedical applications" (No. CZ.02.1.01/0.0/0.0/16_026/0008382). T.S., P.K., and V.L.P.G. also acknowledge the support provided by the Start-up grant of the Experientia Foundation and Dagmar Prochazkova Fund of the University of Chemistry and Technology.

Institutional Review Board Statement: Not applicable.

Informed Consent Statement: Not applicable. 


\section{Data Availability Statement: Not applicable.}

Conflicts of Interest: The authors declare no conflict of interest.

\section{References}

1. Zheng, B.; Gu, G.X. Machine Learning-Based Detection of Graphene Defects with Atomic Precision. Nano-Micro Lett. 2020, $12,181$. [CrossRef]

2. Estrela, P.; Belický, Š.; Katrlík, J.; Tkáč, J. Glycan and Lectin Biosensors. Essays Biochem. 2016, 60, 37-47. [CrossRef] [PubMed]

3. Kveton, F.; Blsakova, A.; Lorencova, L.; Jerigova, M.; Velic, D.; Blixt, O.; Jansson, B.; Kasak, P.; Tkac, J. A Graphene-Based Glycan Biosensor for Electrochemical Label-Free Detection of a Tumor-Associated Antibody. Sensors 2019, 19, 5409. [CrossRef] [PubMed]

4. Damme, E.J.M.V.; Peumans, W.J.; Pusztai, A.; Bardocz, S. Handbook of Plant. Lectins: Properties and Biomedical Applications; John Wiley \& Sons: Hoboken, NJ, USA, 1998; ISBN 978-0-471-96445-2.

5. Bojarová, P.; Křen, V. Sugared Biomaterial Binding Lectins: Achievements and Perspectives. Biomater. Sci. 2016, 4, 1142-1160. [CrossRef]

6. Laaf, D.; Bojarová, P.; Elling, L.; Křen, V. Galectin-Carbohydrate Interactions in Biomedicine and Biotechnology. Trends Biotechnol. 2019, 37, 402-415. [CrossRef]

7. Cordain, L.; Toohey, L.; Smith, M.J.; Hickey, M.S. Modulation of Immune Function by Dietary Lectins in Rheumatoid Arthritis. Br. J. Nutr. 2000, 83, 207-217. [CrossRef]

8. Akkouh, O.; Ng, T.B.; Singh, S.S.; Yin, C.; Dan, X.; Chan, Y.S.; Pan, W.; Cheung, R.C.F. Lectins with Anti-HIV Activity: A Review. Molecules 2015, 20, 648-668. [CrossRef] [PubMed]

9. Eisen, S.; Dzwonek, A.; Klein, N.J. Mannose-Binding Lectin in HIV Infection. Future Virol. 2008, 3, 225-233. [CrossRef] [PubMed]

10. Liu, C.; He, T.; Rong, Y.; Du, F.; Ma, D.; Wei, Y.; Mei, Z.; Wang, Y.; Wang, H.; Zhu, Y.; et al. Association of Mannose-Binding Lectin Polymorphisms with Tuberculosis Susceptibility among Chinese. Sci. Rep. 2016, 6, 36488. [CrossRef]

11. Clausen, T.M.; Sandoval, D.R.; Spliid, C.B.; Pihl, J.; Perrett, H.R.; Painter, C.D.; Narayanan, A.; Majowicz, S.A.; Kwong, E.M.; McVicar, R.N.; et al. SARS-CoV-2 Infection Depends on Cellular Heparan Sulfate and ACE2. Cell 2020, 183, 1043-1057.e15. [CrossRef]

12. Chang, W.-C.; Hartshorn, K.L.; White, M.R.; Moyo, P.; Michelow, I.C.; Koziel, H.; Kinane, B.T.; Schmidt, E.V.; Fujita, T.; Takahashi, K. Recombinant Chimeric Lectins Consisting of Mannose-Binding Lectin and L-Ficolin Are Potent Inhibitors of Influenza A Virus Compared with Mannose-Binding Lectin. Biochem. Pharmacol. 2011, 81, 388-395. [CrossRef]

13. Barondes, S.H.; Cooper, D.N.; Gitt, M.A.; Leffler, H. Galectins. Structure and Function of a Large Family of Animal Lectins. J. Biol. Chem. 1994, 269, 20807-20810. [CrossRef]

14. Merino, J.P.; Serna, S.; Criado, A.; Centeno, A.; Napal, I.; Calvo, J.; Zurutuza, A.; Reichardt, N.; Prato, M. Mass Spectrometry of Carbohydrate-Protein Interactions on a Glycan Array Conjugated to CVD Graphene Surfaces. 2D Mater. 2020, 7, 024003. [CrossRef]

15. Ohno, Y.; Maehashi, K.; Yamashiro, Y.; Matsumoto, K. Electrolyte-Gated Graphene Field-Effect Transistors for Detecting PH and Protein Adsorption. Nano Lett. 2009, 9, 3318-3322. [CrossRef] [PubMed]

16. Gan, T.; Hu, S. Electrochemical Sensors Based on Graphene Materials. Microchim. Acta 2011, 175, 1. [CrossRef]

17. Viswanathan, S.; Narayanan, T.N.; Aran, K.; Fink, K.D.; Paredes, J.; Ajayan, P.M.; Filipek, S.; Miszta, P.; Tekin, H.C.; Inci, F.; et al. Graphene-Protein Field Effect Biosensors: Glucose Sensing. Mater. Today 2015, 18, 513-522. [CrossRef]

18. He, Q.; Wu, S.; Yin, Z.; Zhang, H. Graphene-Based Electronic Sensors. Chem. Sci. 2012, 3, 1764-1772. [CrossRef]

19. Justino, C.I.L.; Gomes, A.R.; Freitas, A.C.; Duarte, A.C.; Rocha-Santos, T.A.P. Graphene Based Sensors and Biosensors. TrAC Trends Anal. Chem. 2017, 91, 53-66. [CrossRef]

20. Nag, A.; Mitra, A.; Mukhopadhyay, S.C. Graphene and Its Sensor-Based Applications: A Review. Sens. Actuators Phys. 2018, 270, 177-194. [CrossRef]

21. Huang, H.; Su, S.; Wu, N.; Wan, H.; Wan, S.; Bi, H.; Sun, L. Graphene-Based Sensors for Human Health Monitoring. Front. Chem. 2019, 7. [CrossRef]

22. Schwefel, D.; Maierhofer, C.; Beck, J.G.; Seeberger, S.; Diederichs, K.; Möller, H.M.; Welte, W.; Wittmann, V. Structural Basis of Multivalent Binding to Wheat Germ Agglutinin. J. Am. Chem. Soc. 2010, 132, 8704-8719. [CrossRef]

23. Kovaříček, P.; Vrkoslav, V.; Plšek, J.; Bastl, Z.; Fridrichová, M.; Drogowska, K.; Kalbáč, M. Extended Characterization Methods for Covalent Functionalization of Graphene on Copper. Carbon 2017, 118, 200-207. [CrossRef]

24. Bojarová, P.; Chytil, P.; Mikulová, B.; Bumba, L.; Konefał, R.; Pelantová, H.; Krejzová, J.; Slámová, K.; Petrásková, L.; Kotrchová, L.; et al. Glycan-Decorated HPMA Copolymers as High-Affinity Lectin Ligands. Polym. Chem. 2017, 8, 2647-2658. [CrossRef]

25. Bojarová, P.; Kulik, N.; Hovorková, M.; Slámová, K.; Pelantová, H.; Křen, V. The $\beta$-N-Acetylhexosaminidase in the Synthesis of Bioactive Glycans: Protein and Reaction Engineering. Molecules 2019, 24, 599. [CrossRef]

26. Hallam, T.; Berner, N.C.; Yim, C.; Duesberg, G.S. Strain, Bubbles, Dirt, and Folds: A Study of Graphene Polymer-Assisted Transfer. Adv. Mater. Interfaces 2014, 1, 1400115. [CrossRef]

27. Kalbac, M.; Frank, O.; Kavan, L. The Control of Graphene Double-Layer Formation in Copper-Catalyzed Chemical Vapor Deposition. Carbon 2012, 50, 3682-3687. [CrossRef]

28. Kovaříček, P.; Bastl, Z.; Valeš, V.; Kalbáč, M. Covalent Reactions on Chemical Vapor Deposition Grown Graphene Studied by Surface-Enhanced Raman Spectroscopy. Chem. Eur. J. 2016, 22, 5404-5408. [CrossRef] 
29. Plšek, J.; Kovaříček, P.; Valeš, V.; Kalbáč, M. Tuning the Reactivity of Graphene by Surface Phase Orientation. Chem. Eur. J. 2017, 23, 1839-1845. [CrossRef] [PubMed]

30. Langer, R.; Zaoralová, D.; Medved', M.; Banáš, P.; Błoński, P.; Otyepka, M. Variability of C-F Bonds Governs the Formation of Specific Structural Motifs in Fluorinated Graphenes. J. Phys. Chem. C 2019, 123, 27896-27903. [CrossRef]

31. Hong, V.; Presolski, S.I.; Ma, C.; Finn, M.G. Analysis and Optimization of Copper-Catalyzed Azide-Alkyne Cycloaddition for Bioconjugation. Angew. Chem. Int. Ed. 2009, 48, 9879-9883. [CrossRef] [PubMed]

32. Rafiee, J.; Mi, X.; Gullapalli, H.; Thomas, A.V.; Yavari, F.; Shi, Y.; Ajayan, P.M.; Koratkar, N.A. Wetting Transparency of Graphene. Nat. Mater. 2012, 11, 217-222. [CrossRef] [PubMed]

33. Kozbial, A.; Zhou, F.; Li, Z.; Liu, H.; Li, L. Are Graphitic Surfaces Hydrophobic? Acc. Chem. Res. 2016, 49, 2765-2773. [CrossRef]

34. She, C.Y.; Dinh, N.D.; Tu, A.T. Laser Raman Scattering of Glucosamine, N-Acetylglucosamine, and Glucuronic Acid. Biochim. Biophys. Acta Gen. Subj. 1974, 372, 345-357. [CrossRef]

35. Bansil, R.; Yannas, I.V.; Stanley, H.E. Raman Spectroscopy: A Structural Probe of Glycosaminoglycans. Biochim. Biophys. Acta Gen. Subj. 1978, 541, 535-542. [CrossRef]

36. Carron, K.T.; Hurley, L.G. Axial and Azimuthal Angle Determination with Surface-Enhanced Raman Spectroscopy: Thiophenol on Copper, Silver, and Gold Metal Surfaces. J. Phys. Chem. 1991, 95, 9979-9984. [CrossRef]

37. Moullier, P.; Daveloose, D.; Leterrier, F.; Hoebeke, J. Comparative Binding of Wheat Germ Agglutinin and Its Succinylated Form on Lymphocytes. Eur. J. Biochem. 1986, 161, 197-204. [CrossRef] [PubMed] 